\title{
Modified Fast and Enhanced Exemplar based Inpainting Algorithm for Solving Unknown Row Filling Problem
}

\author{
Manali Desai \\ U \& P U Patel Department of \\ Computer Engineering \\ Chandubhai S Patel Institute of \\ Technology \\ Charusat,Change,Gujarat,India
}

\begin{abstract}
Inpainting algorithm is used to fill missing information in an image. This paper proposes modified fast and enhanced exemplar based inpainting algorithm for solving unknown row filling problem. This technique is modification in updating criteria in fast and enhanced exemplar based inpainting algorithm. Our experimental results show that proposed scheme gives better result for unknown row filling problem.
\end{abstract}

\section{General Terms}

Image Processing, Image, Inpainting algorithm

\section{Keywords}

Unknown row filling, Exemplar based Inpainting

\section{INTRODUCTION}

There are many inpainting algorithms. We can categorized it into three category.PDE or convolution based [7],[8] based on classification of image blocks into texture and structure,[9],[10]exemplar based algorithms.[1],[2],[3],[4],[5],[6].

The exemplar-based inpainting takes an exemplar and generates additional content based on that exemplar to create much more content than is contained in the exemplar. Blur is not seen when filling large area using exemplar based inpainting algorithm which is seen when filling large area using PDE based inpainting algorithm. This type of algorithm incorporates advantages of both image inpainting and texture synthesis. So, our focus is on exemplar based inpainting algorithms. Many researchers have proposed a large variety of exemplar based image inpainting algorithms to restore the structure and texture of damaged images.

This paper gives solution for unknown row filling problem found in fast and enhanced algorithm for exemplar based. In the experiment we show that our approach is superior over earlier approach. Rest of this paper is organized as follow. Literature review and comparative study of various exemplar based inpainting algorithm is shown in section 2. Analysis of existing fast and enhanced algorithm for exemplar based is given in section 3. Design of proposed algorithm is given in section 4. Results are shown in section 5. Section 6 shows conclusion and future extension.

\section{LITERATURE REVIEW}

\subsection{Exemplar Based Inpainting Algorithm}

Common steps seen in various exemplar based inpainting algorithm are as follows:

1. Mark the inpainting region
2. Decide boundary of missing region

3. Get the patch from boundary of missing information (patch which contains missing as well as known information.)

4. Select patch based on priority. Find another patch from known area which is similar with selected patch. Metric for finding similar patch may be different with different exemplar based algorithms.

5. Update missing information in selected patch from found similar patch Mark selected patch region as known region.

6. Repeat steps 3 to step 6 until whole inpainted region is not filled. [1]

We call step 3 as priority criteria, step 4 as searching criteria and step 5 and step 6 as updating criteria. Various exemplar based algorithm has its own priority criteria and searching criteria. We have done comparative analysis based on these criteria.

\subsection{Exemplar Based Inpainting Algorithm Variations}

Alexander Wong and Jeff Orchard presented a non-local means approach to exemplar-based inpainting [3]. An image with redundant content could have several samples that could be combined to form a more robust estimate of the missing information. In [3] authors proposed that improved inpainting quality can be achieved using nonlocal information from multiple samples within the image. The relative contribution of each sample to the reconstruction of a target pixel is determined using a weighted similarity function and aggregated to form the missing information.

In [4] Robust Algorithm for Exemplar Based Inpainting is presented, their main contribution is generic priority function to provide robust performance.

Criminisi's [2] contribution in exemplar based algorithm is that they have explored a fill order which explicitly encourages propagation of linear structure (together with texture), the default favorite has been the "onion peel" strategy. Avoid "over-shooting" artifacts that occur when image edges are allowed to grow indefinitely. The goal was to find good balance between the propagation of structured regions and that of textured regions the algorithm proposed algorithm achieves such a balance by combining the structure "push" with a confidence term that tends to reduce sharp inshooting appendices in the contour of the target region.

Anupam [1] give solution to the problem, if two or more patch has same mean square error, so to decide patch which is most suitable for the patch to be filled is done by calculating variance. In order to reduce computational complexity instead of searching in whole image, reduced search area is 
proposed.

In [5] novel priority scheme is designed to assign priority and guide the filling-in order, which is a crucial step for the performance of the inpainted results. New data term is introduced based on calculation of variation of variance of neighboring patch.

In [6] Authors extend an exemplar-based image inpainting method by incorporating Bézier curves to construct missing edge information. Mean shift segmentation is used to extract contours of each region. After the structure information of an image is determined, destroyed contours will be connected in curve fitting process and damaged regions will be inpainted by using exemplar-based inpainting method. They introduce a new data term, based on edge information and a new concept of patch template, to make sure that the best structural similarity is achieved.

\subsection{Comparative Analysis of Exemplar Based Inpainting Algorithm}

Various search criteria in various exemplar based inpainting algorithm are categorized as one or more patch which uses more than one patch as name suggest, Patch template which uses neighboring pixels of patch, Reduced search are searches for similar patch in nearby region of inpainting region instead of searching in whole image. Priority calculation in various exemplar based inpainting algorithm is categorized as Isophote driven which uses confidence and data terms in its calculation for considering texture and structure information. Confidence term represents reliable information surrounding. Data term is function of the strength of isophotes hitting the front, Edge driven priority add information of edge, Variation of variance priority scheme as the name suggest emphasize on

Table 1 Comparative Analysis of Modifications in Exemplar based Inpainting Algorithm

\begin{tabular}{|c|c|c|c|c|c|c|}
\hline \multirow[t]{2}{*}{ Algorithm } & \multicolumn{3}{|c|}{$\begin{array}{l}\text { Modification related to } \\
\text { search }\end{array}$} & \multicolumn{3}{|c|}{$\begin{array}{l}\text { Modification related to } \\
\text { priority calculation }\end{array}$} \\
\hline & $\begin{array}{l}\text { One } \\
\text { or } \\
\text { more } \\
\text { patch }\end{array}$ & $\begin{array}{c}\text { Patch } \\
\text { templ } \\
\text { ate }\end{array}$ & $\begin{array}{c}\text { Reduce } \\
\text { d } \\
\text { search } \\
\text { area }\end{array}$ & $\begin{array}{l}\text { Isop } \\
\text { hote } \\
\text { driv } \\
\text { en }\end{array}$ & $\begin{array}{c}\text { Variat } \\
\text { ion of } \\
\text { varian } \\
\text { ce }\end{array}$ & $\begin{array}{c}\text { Edge } \\
\text { drive } \\
n\end{array}$ \\
\hline Criminisi & & & & YES & & \\
\hline A.Wong & YES & & & & & \\
\hline W.Cheng & & & & YES & & \\
\hline $\begin{array}{l}\text { Qing } \\
\text { Zhang }\end{array}$ & & & & & YES & \\
\hline Jason & & YES & & & & YES \\
\hline Anupam & & & YES & & & \\
\hline
\end{tabular}

variance calculation.

Table 2 Limitations in Exemplar based inpainting algorithms

\begin{tabular}{|l|l|}
\hline Algorithm & Limitations \\
\hline Criminisi & $\begin{array}{l}\text { It cannot handle curve structure. It doesn't } \\
\text { consider depth information in image. }\end{array}$ \\
\hline A.Wong & $\begin{array}{l}\text { It cannot handle complex geometrical } \\
\text { structure }\end{array}$ \\
\hline W.Cheng & $\begin{array}{l}\text { It cannot handle complex geometrical } \\
\text { structure }\end{array}$ \\
\hline Qing Zhang & $\begin{array}{l}\text { It cannot handle complex geometrical } \\
\text { structure }\end{array}$ \\
\hline Jason & $\begin{array}{l}\text { Efficiency of inpainting depends upon } \\
\text { mean shift segmentation algorithm and } \\
\text { also on Bezier curve.It cannot handle } \\
\text { complex geometrical structure. }\end{array}$ \\
\hline Anupam & $\begin{array}{l}\text { Results are more promising than } \\
\text { Criminisi's approach. But it takes 52378 } \\
\text { seconds to reconstruct image of 60492 } \\
\text { pixels with 14.62\% area to be removed. }\end{array}$ \\
\hline
\end{tabular}

Limitations of various exemplar based inpainting algorithm is also analyzed. Our chosen algorithm is[1] fast and enhanced exemplar based inpainting algorithm as it can reconstruct image are in less computation time and also gives solution for multiple similar mean square error patch. We are extending this[1] algorithm.

\section{ANALYSIS OF EXSITING ALGORITHM}

Our chosen algorithm is[1] fast and enhanced exemplar based inpainting algorithm as it can reconstruct image are in less computation time and also gives solution for multiple similar mean square error patch. We are extending this [1] algorithm.

\subsection{Various Parameters}

Various parameters of Fast and Enhanced Exemplar based image inpainting are Alpha $\alpha$, Beta $\beta$, Omega $\omega$, window size w. $\alpha$ and $\beta$ are component weights for confidence and data term respectively. $\omega$ is regularizing factor for controlling the curve smoothness. Using this confidence term the value of the confidence term is regularized to $[\omega, 1]$. Window size $w$ denotes patch size. 


\subsection{Examining Nature}

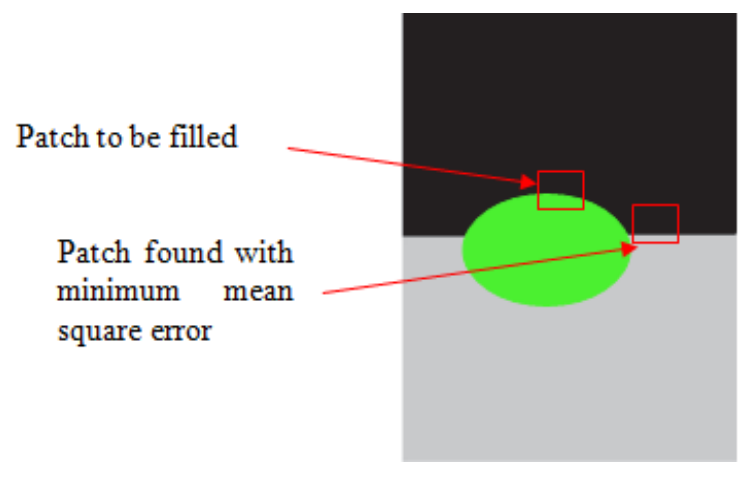

Fig 1 Unknown Row Filling Problem

This method can reconstruct large image area without blur. It is computationally expensive but takes less computation time than Criminisi's approach. Searching best exemplar takes more computational time and mean square error calculation for one patch does not depends upon another patch hence they can be considered as independent task which shows parallel behavior. Filling order is critical, it is greedy algorithm. We have identified unknown row filling behavior.

We have investigated one case of incorrect filling in the image. We denote patch to be filled as $\Psi \mathrm{p}$ and best patch match found by $\Psi$ q. after applying best exemplar search in fast and enhanced exemplar based inpainting algorithm, patch with minimum error is found. There is no knowledge for filling in lower rows of patch $\Psi \mathrm{p}$. Minimum error is found by considering upper rows of $\Psi \mathrm{p}$ and $\Psi \mathrm{q}$. Hence it will produce wrong filling. We will term this problem as "Unknown row filling problem" throughout this paper.

\subsubsection{Solution for Unknown Row Filling Problem} We have thought various solutions for solving "unknown row filling problem"

\subsubsection{Modify priority calculation}

We should modify priority function such that patch suffering from unknown row filling problem should not get higher priority. But as by lowering priority of patch suffering from unknown row filling problem other patch having lower priority get higher priority, which may propagate wrong information. When designing priority function, all things mentioned above should be taken care.

\subsubsection{Modify searching criteria:}

When searching for best exemplar match, not only consider information within patch only, but also consider neighboring information outside of patch for matching. For that we have to consider up to which extend in neighborhood also there is missing information. If information is missing in neighborhood also, than there won't be any benefit of searching by considering that neighborhood. It is somewhat similar concept as patch template given in [28] but here difference is they are setting value of neighborhood in algorithm and our idea is to make algorithm automatically decide it.

\subsubsection{Modify updating criteria:}

If found patch with minimum mean square error is suffering from "unknown row filling problem", update information of remaining raw which are not suffering from this problem. By doing so valid portion of found patch will be copied correctly and invalid portion will be ignored, hence it will not propagate wrong information in image. So size of patch which will be filled is decided adaptively during filling. So, we will term proposed method as "Adaptive size filling method". We have worked on this proposed idea.

\section{DESIGN OF PROPOSED ALGORITHM}

Here design of this proposed algorithm is presented. It includes pseudo code design for proposed algorithm.

1) For each pixel $p$ in inpainting region, $p=0$ (This is performed separately from inpainting process.)

2) Find the boundary of target region.

3) Divide the marked region in to patches and assign priority to each patch.

4) Check for patch in the list, (Modifications in original algorithm).If any row is not suffering from "Unknown row filling problem" Release it from list.

5) Patch to be inpainted =MaxPriority (patch) and patch not in list(Modifications in original algorithm)

6) Find the patch from the image which best matches the selected patch. This can be done by finding suitable error metric. Mean Squared Error is chosen $\mathrm{MSE}=\sum\left(\mathrm{f}_{\mathrm{x}, \mathrm{y}} \mathrm{g}_{\mathrm{x}, \mathrm{y}}\right)^{2} / \mathrm{N}$

$\mathrm{N}$ is the total number of elements in the patch

fx,y represents element of the patch.

Gx,y represents element of the patch for which MSE is calculated.

7) Update image information.(Modifications in original algorithm)Check if found patch is suffering from unknown row filling problem, If it is not suffering from this problem

Update the image information.

Else

Update only those pixels which are not in the row due to which patch suffers from unknown. Put that patch in list.

\section{EXPERIMENTS AND RESULTS}

To verify the impact of modification in updating criteria, we performed test on several images and compared so-obtained 
results with conventional approach. Several of the images are taken from various literature and we cite appropriate paper whenever possible. Patch size is taken same as in original fast and enhanced exemplar based inpainting algorithm (9x9).
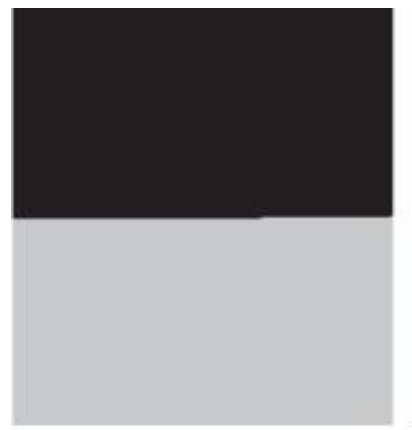

a

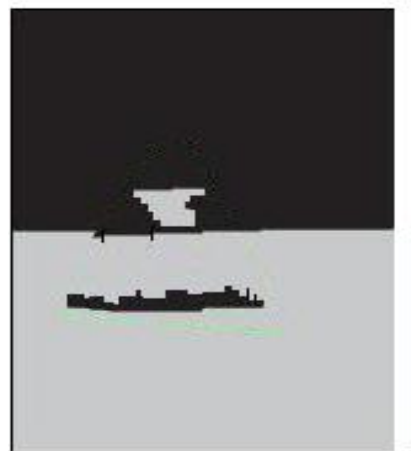

c

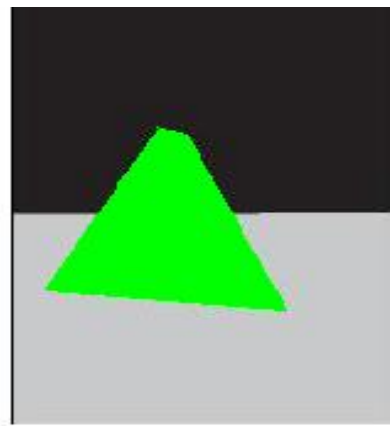

b

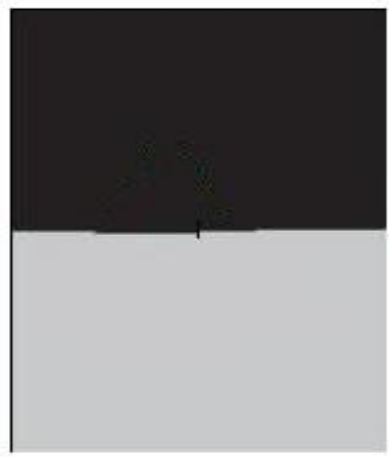

d
Fig 2 EXPERIMENT1: Comparison with Original Fast and enhanced algorithm for exemplar based. $a$ is original image, $b$ is marked with inpainting region, $c$ is result by original algorithm, $d$ is results by our proposed method.

\subsection{Experiments on Static Image}

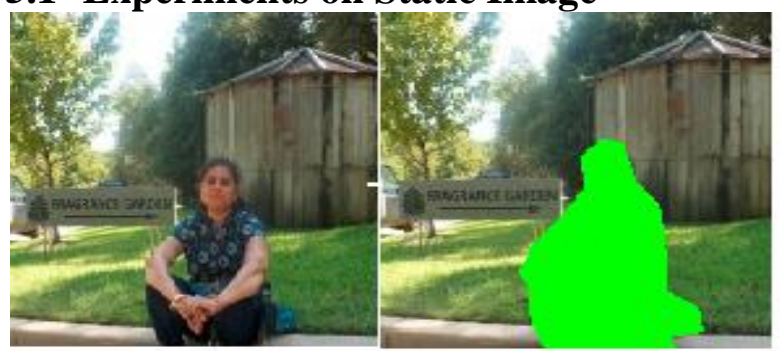

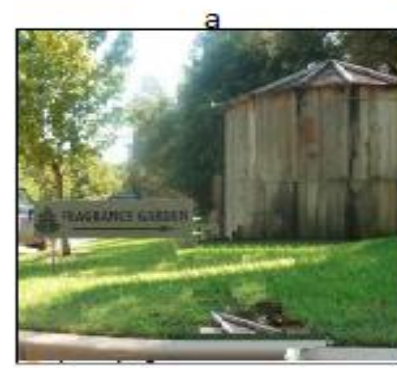

C

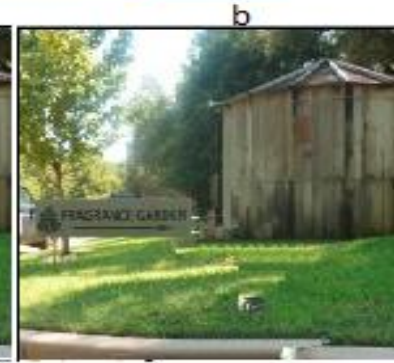

d
Fig 3 EXPERIMENT2: Comparison with Original Fast and enhanced algorithm for exemplar based on images with static objects. $a$ is original images, $b$ is marked with inpainting region, $c$ is results by original algorithm, $d$ is results by our proposed method.
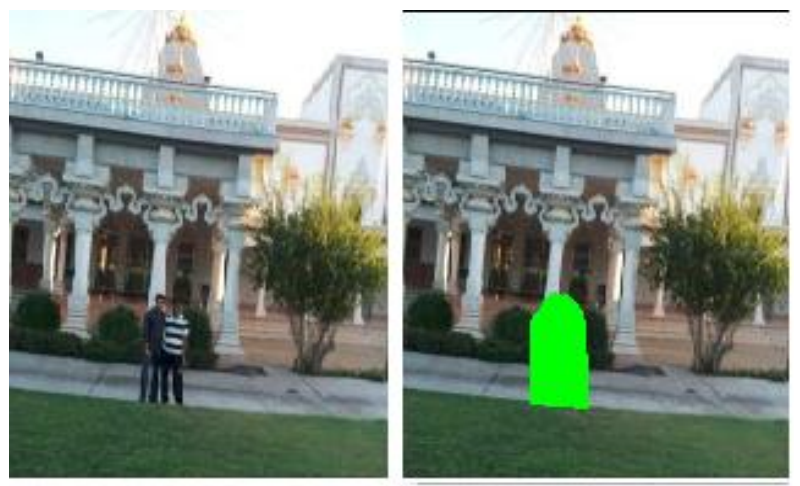

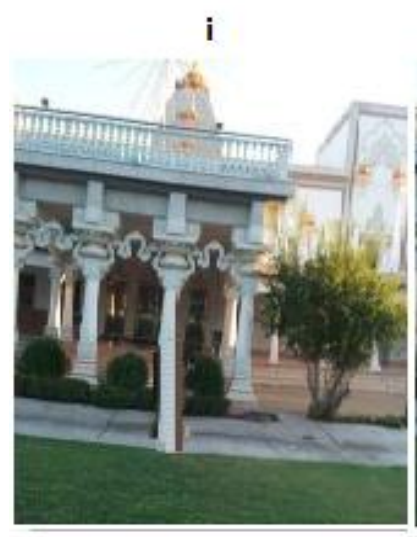

k

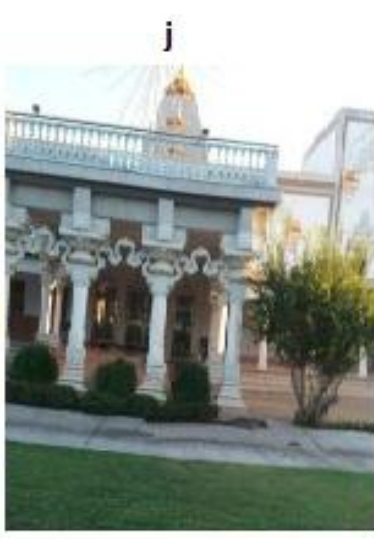

I
Fig 4 EXPERIMENT3: Comparison with Original Fast and enhanced algorithm for exemplar based on images with static objects. $i$ is original images, $j$ is marked with inpainting region, $k$ is results by original algorithm, $l$ is results by our proposed method.
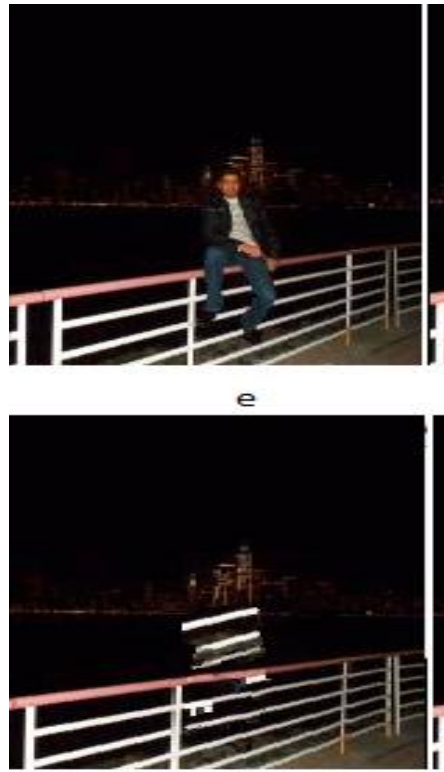

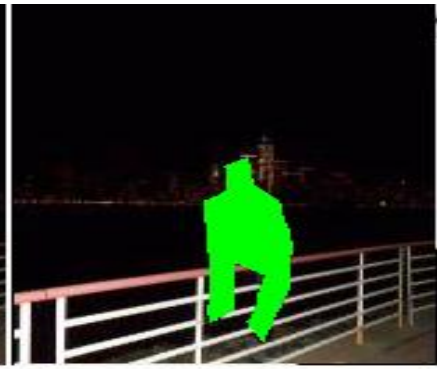

$f$

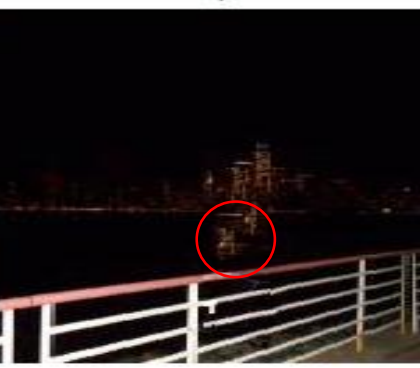

Fig 5 EXPERIMENT4: Comparison with Original Fast and enhanced algorithm for exemplar based on images with static objects. $e$ is original images, $f$ is marked with inpainting region, $g$ is results by original algorithm, $h$ is results by our proposed method. 
In Figure 3 we have tried to remove woman from photograph. With original fast and enhanced exemplar based inpainting algorithm we can see some artificial feature is added as in Figure 3 (c), We have removed some of unwanted feature as shown in Figure3 (d) by applying solution to unknown row filling problem. There is need to identify problem due to which it creates some wrong unwanted feature in order to even more improve this algorithm so to achieve acceptable solution. Figure 4 and Figure 5 also shows improvement in result by our proposed method. Figure 5(h) shows wrong information marked by red circle due to lack of the knowledge about background. White strips wrongly created in Figure $5(\mathrm{~g})$ are removed by applying solution to unknown row filling problem.

\subsection{Experiments on Moving Objects}

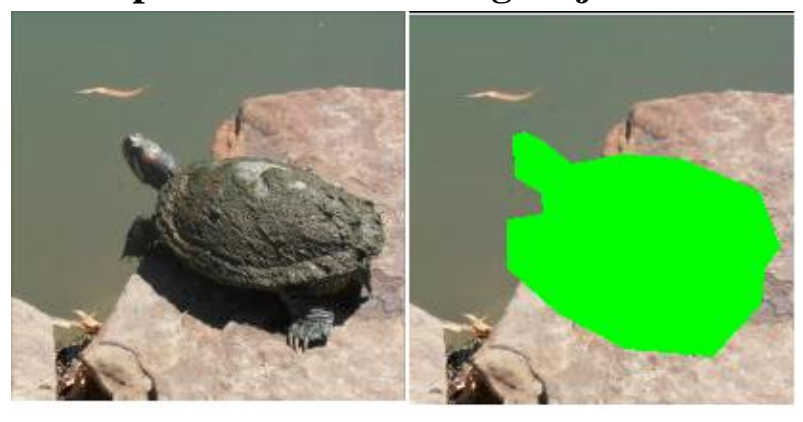

a

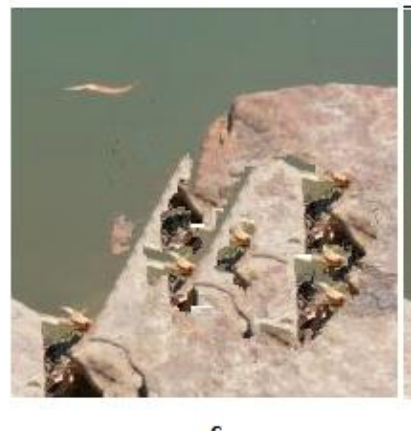

b

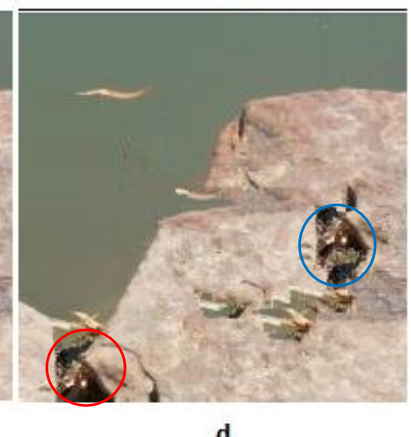

Fig 6 EXPERIMENT5: Comparison with Original Fast and enhanced algorithm for exemplar based with images having static objects. $a$ is original image, $b$ is marked with inpainting region, $c$ is result by original algorithm, $d$ is results by our proposed method.

In Figure 6 we have tried to remove moving turtle, We have solved the problem of unknown row filling in Figure 6(d), but still it suffers from edge continuity problem. It is clear from result that it copied background information marked by red circle in place of blue circle.

\section{CONCLUSION AND FUTURE EXTENSION}

We identified unknown row filling problem in filling problem in fast and enhanced algorithm for exemplar based and proposed method to solve it .Proposed improvement solves the problem of unknown row filling and better result than original algorithm for fast and enhanced exemplar based inpainting algorithm [1]

But still experiments with images shows that it suffers from other problems due to which it adds some unwanted wrong information from background in the image. Future work is knowledge of background information which includes edge continuity, neighboring object information, knowledge of distinction between two different objects in image or between object and background should be added when copying patch to make this algorithm intelligent.

\section{ACKNOWLEDGMENTS}

I would like to thank my guide Prof Amit Ganatra for providing guidance for carrying out research work in my dissertation. I would also like to thank my husband Mr Ronak Vashi and my mother Mrs Nayana Desai for encouraging me and helping me and also my brother Mr Dhaval Desai for providing me Test images.

\section{REFERENCES}

[1] Anupam,Pulkit Goyal,Sapan Diwakar"Fast and Enhanced Algorithm for Exemplar based Image Inpainting”, Fourth Pacific Rim Symposium on Image and Vedio Technology,2010

[2] A. Criminisi, P. P'erez and K. Toyama “ Region Filling and Object Removal by Exemplar Based Image Inpainting”, IEEE Transaction on Image Processing,vol13,no.9,SEP 2004

[3] A. Wong and J. Orchard, "A nonlocal-means approach to exemplar based inpainting," presented at the IEEE Int. Conf. Image Processing, 2008

[4] W. Cheng, C. Hsieh, S. Lin, C. Wang, and J. Wu, "Robust algorithm for exemplar-based image inpainting," in Proceedings of International Conference on Computer Graphics, Imaging and Visualization, 2005, pp. 64-69

[5] Qing Zhang and Jiajun Lin, "Exemplar-based Image Inpainting Using Color Distribution Analysis", Journal of Information Science and Engineering,2011

[6] Jason C. Hung, Chun-Hong Hwang, Yi-Chun Liao, Nick C. Tang and Ta-Jen Chen, "Exampler based inpainting based on structure construction", JOURNAL OF SOFTWARE, VOL. 3, NO. 8, NOVEMBER 2008

[7] Bertalmio, M, Sapiro, G., Caselles, V., Ballester, C. "Image Inpainting". SIGGRAPH 2000, pages 417-424.

[8] Manuel M.Oliveira,Brian Bowen,Richard McKenna,YuSung Chang "Fast Digital Image Inpainting”VIIP 2001

[9] S. D Rane, J. Remus, and G. Sapiro, "Wavelet Domain Reconstruction of Lost Blocks in Wireless Image Transmission and Packet Switched Network," in Proc.Image Processing, 2002, pp. I-309 - I-312

[10] S. D. Rane, G. Sapiro, and M. Bertalmio, "Structure and Texture Filling -In of Missing Image Blocks in Wireless Transmission and Compression Application," IEEE Trans. on Image Processing, vol.12, no.3, pp.296-303, March2003. 